\title{
An attenuated strain of Bacillus anthracis (CDC 684) has a large chromosomal inversion and altered growth kinetics
}

Richard T Okinaka ${ }^{1,2^{*}}$, Erin P Price ${ }^{1}$, Spenser R Wolken ${ }^{1}$, Jeffrey M Gruendike ${ }^{1}$, Wai Kwan Chung ${ }^{1}$, Talima Pearson ${ }^{1}$, Gary Xie ${ }^{2}$, Chris Munk ${ }^{2}$, Karen K Hill2 ${ }^{2}$, Jean Challacombe ${ }^{2}$, Bruce E Ivins ${ }^{3}$, James M Schupp ${ }^{4}$, Stephen M Beckstrom-Sternberg ${ }^{1,4}$, Arthur Friedlander ${ }^{3}$ and Paul Keim ${ }^{1,2,4}$

\begin{abstract}
Background: An isolate originally labeled Bacillus megaterium CDC 684 was found to contain both pXO1 and pXO2, was non-hemolytic, sensitive to gamma-phage, and produced both the protective antigen and the poly-Dglutamic acid capsule. These phenotypes prompted Ezzell et al., (J. Clin. Microbiol. 28:223) to reclassify this isolate to Bacillus anthracis in 1990.

Results: We demonstrate that despite these $B$. anthracis features, the isolate is severely attenuated in a guinea pig model. This prompted whole genome sequencing and closure. The comparative analysis of CDC 684 to other sequenced B. anthracis isolates and further analysis reveals: a) CDC 684 is a close relative of a virulent strain, Vollum A0488; b) CDC 684 defines a new B. anthracis lineage (at least 51 SNPs) that includes 15 other isolates; c) the genome of CDC 684 contains a large chromosomal inversion that spans $3.3 \mathrm{Mbp}$; d) this inversion has caused a displacement of the usual spatial orientation of the origin of replication (ori) to the termination of replication (ter) from $180^{\circ}$ in wild-type B. anthracis to $120^{\circ}$ in CDC 684 and e) this isolate also has altered growth kinetics in liquid media.

Conclusions: We propose two alternative hypotheses explaining the attenuated phenotype of this isolate. Hypothesis 1 suggests that the skewed ori/ter relationship in CDC 684 has altered its DNA replication and/or transcriptome processes resulting in altered growth kinetics and virulence capacity. Hypothesis 2 suggests that one or more of the single nucleotide polymorphisms in CDC 684 has altered the expression of a regulatory element or other genes necessary for virulence.
\end{abstract}

\section{Background}

Attenuated strains of Bacillus anthracis have played a major role in the development of vaccines and our understanding of anthrax. Early work by Pasteur and Greenfield [1,2] capitalized upon strains missing one of the mega-plasmids (pXO1), which resulted in attenuation. This enabled the development of the first bacterial disease to be prevented through the use of an attenuated live vaccine. This early work was improved by Sterne [3] through the development of an attenuated strain missing the second mega-plasmid (pXO2), but retaining the

\footnotetext{
* Correspondence: Richard.Okinaka@nau.edu

${ }^{1}$ Center for Microbial Genetics and Genomics, Northern Arizona University, Flagstaff, AZ 86011, USA

Full list of author information is available at the end of the article
}

toxin producing genes on pXO1 as antigens for immune response. In recent years, avirulent strains have been subjected to extensive DNA sequencing to understand these plasmids, their virulence genes and to generate hypotheses for attenuation mechanisms [4-11]. Conversely $B$. cereus strains that have acquired the known $B$. anthracis mega-plasmids and anthrax-like virulence properties remain an enigma and are also worthy of further study to understand how this pathogen interacts with its host [12-14].

An isolate from the Centers for Disease Control (CDC) originally identified as B. megaterium, CDC 684/ NRRL-349S/NRS 234 (herein called CDC 684), was being used as an avirulent outgroup control in experiments with $B$. anthracis [15]. However, this particular
C Biomed Central

() 2011 Okinaka et al; licensee BioMed Central Ltd. This is an Open Access article distributed under the terms of the Creative Commons Attribution License (http://creativecommons.org/licenses/by/2.0), which permits unrestricted use, distribution, and reproduction in any medium, provided the original work is properly cited. 
isolate shares key phenotypic traits with $B$. anthracis such as non-hemolytic on blood agar, production of protective antigen and the poly-D-glutamic acid capsule, and sensitivity to gamma bacteriophage. Because these features are all hallmark phenotypes for B. anthracis, Ezzell et al. [15] reclassified this isolate as B. anthracis despite the observation that $\mathrm{CDC} 684$ did not react with monoclonal antibodies to a specific polysaccharide present in B. anthracis. Subsequent animal testing of this isolate showed it to be severely attenuated in guinea pigs, in contrast to wild-type B. anthracis (See results, Attenuation of CDC 684). However, the underlying mechanism behind this attenuated virulence phenotype remained unknown. The advent of massively parallel whole genome sequencing (WGS) provides an opportunity to examine the complete genetic component of CDC 684 for clues that might bear on this problem.

This report provides a description of the WGS, assembly and annotation of the B. anthracis CDC 684 isolate. We include analysis that: a) demonstrates that the genome of CDC 684 belongs to a specific $B$. anthracis clade; b) identifies 51 single nucleotide polymorphisms (SNP) that are unique to the genome of this isolate; c) describes the details of a large chromosomal inversion; d) demonstrates that CDC 684 has altered growth kinetics in culture and e) proposes two alternative and testable hypotheses that could explain the attenuated phenotype for CDC 684 .

\section{Results}

\section{Attenuation of CDC 684}

The discovery that CDC 684 was not a $B$. megaterium strain but was rather $B$. anthracis, based on shared phenotypic features, prompted the use of the guinea pig model to determine its virulence. In a pilot experiment, groups of four guinea pigs injected i.m. with CDC 684 spores at doses of $114,1,145$, and $11,450 \mathrm{cfu} / \mathrm{mL}$ survived. These groups were then injected four days later with $1.29 \times 10^{5}, 1.29 \times 10^{6}$ and $1.29 \times 10^{7} \mathrm{cfu} / \mathrm{mL}$, respectively, and again all survived. By comparison these identical spore preparation and treatment conditions produced $\mathrm{LD}_{50}$ values for the virulent Ames and Vollum-1B strains of 175 and 306 spores respectively in the guinea pig model $[16,17]$.

This lack of lethality indicated that CDC 684 is significantly attenuated. In a second experiment to confirm attenuation, 10 guinea pigs injected i.m. with $1 \times 10^{8}$ $\mathrm{cfu} / \mathrm{mL}$ CDC 684 spores all survived. These results confirm that CDC 684 is highly attenuated with an $\mathrm{LD}_{50}$ of $>1 \times 10^{8}$ spores in the guinea pig model.

\section{WGS of CDC 684}

The CDC 684 genome has been recently sequenced and assembled to closure at Los Alamos National
Laboratory/J. Craig Venter Institute and is available on the NCBI Genome database [GenBank: CP001215.1]. The chromosome is 5,230,115 bp, pXO1 [GenBank: CP001216] is 181,773 bp and pXO2 [GenBank: $\mathrm{CP} 001214]$ is $94,875 \mathrm{bp}$.

\section{Phylogenetic placement of CDC 684}

The use of comparative WGS defined an extremely conserved and accurate phylogenetic SNP tree for $B$. anthracis based on the analysis of 1,000 SNPs in 26 diverse isolates [18]. This analysis resulted in the hypothesis that only a few selected SNPs at key positions along five branches were needed to accurately place all $B$. anthracis isolates into one of 12 sub-clades. This notion was shown to be accurate when 13 canSNPs were subsequently used to accurately place more than $1,000 \mathrm{~B}$. anthracis isolates into one of these $12 \mathrm{sub}-$ clades [19]. In silico canSNP typing showed that CDC 684 falls along the lineage created by $B$. anthracis Vollum (A0488; [GenBank: ABJC00000000]). This sequenced Vollum strain is presumed to be a close relative of the British isolate that was tested as a biological weapon on Gruinard Island, Scotland, in the 1940s [20].

The close phylogenetic relationship between CDC 684 and Vollum demonstrates that CDC 684 belongs to a highly virulent $B$. anthracis lineage. We were therefore interested in further determining the degree of relatedness between Vollum and CDC 684, given the marked differences in virulence between these two strains. An initial comparative in silico analysis of Ames Ancestor [GenBank: AE017334], CDC 684 and Vollum WGS uncovered $~ 390$ SNP differences distinct from Ames Ancestor but common (i.e., derived) in both the CDC 684 and Vollum genomes. These results are consistent with other whole genome SNP comparisons of $128 \mathrm{~B}$. anthracis isolates that suggest that the SNP genetic distance between Ames and Vollum is approximately 400 SNPs [Pearson, Schupp, Ravel and Keim, unpublished data].

Preliminary analysis of 30 SNPs that phylogenetically reside along a terminal position on the Vollum branch indicated that there were at least 10 new nodes along this branch, of which $>100$ Vollum-like isolates reside [Chung, Pearson and Keim, unpublished data]. In silico analysis of 10 new canSNPs along this branch indicated that CDC 684 was not in the terminal node created by the sequenced Vollum strain, but rather was located in a node midway between the sequenced strain and a branch point defined by the initial analysis of $100 \mathrm{Vol}-$ lum-like strains, Figure 1.

\section{CDC specific SNPs}

This analysis also demonstrated that CDC 684 possessed 51 SNPs that appeared to be unique to this isolate. There 


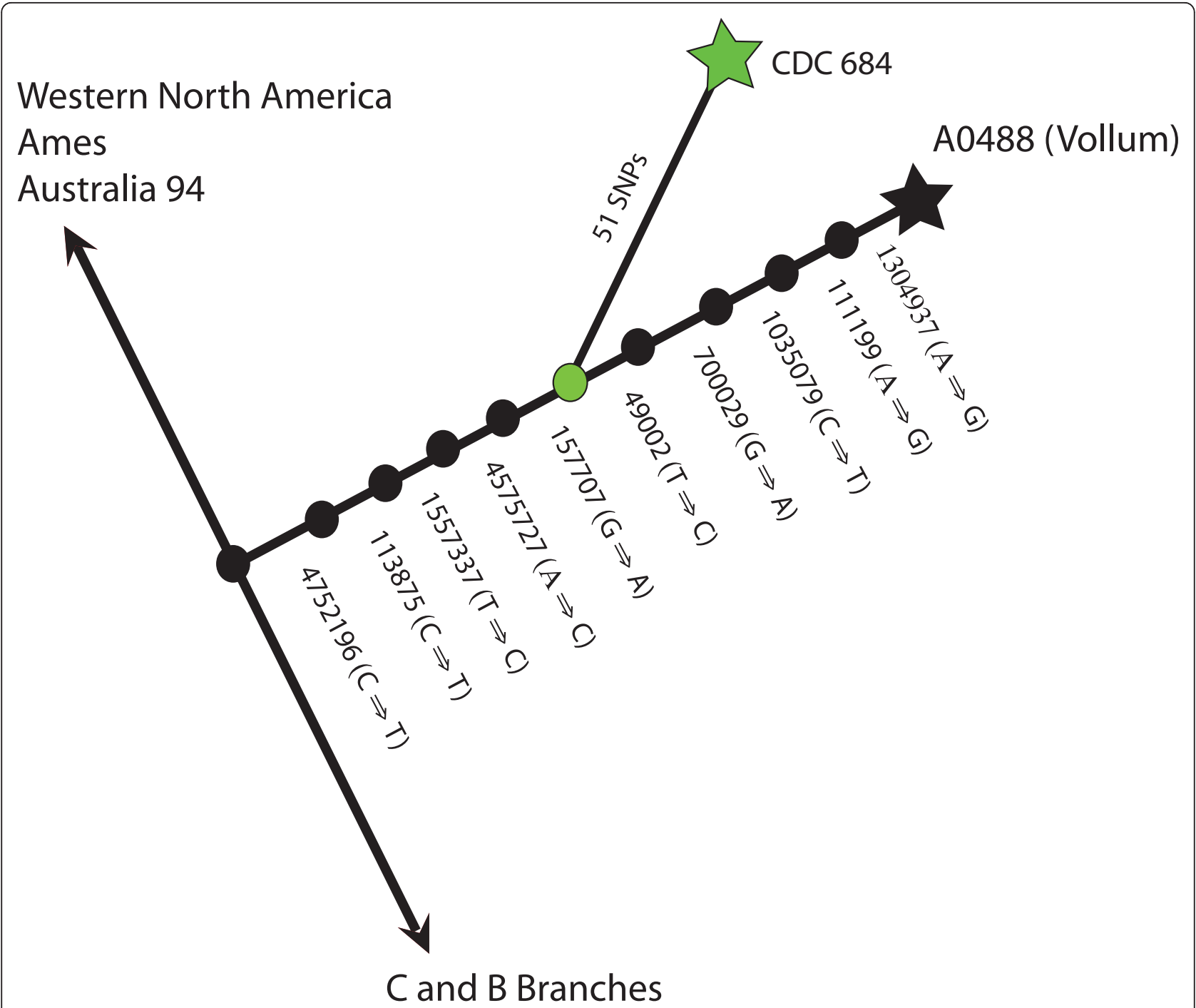

Figure 1 Location of CDC 684 on the B. anthracis phylogenetic tree. Genotypic analysis of 30 SNPs in 100 isolates from the original Vollum node (Chung, Pearson and Keim, unpublished data) created 10 new collapsed branch points (nodes) along the Vollum sub-lineage (black circles). The positions of 10 new canSNPs are designated by the ancestral to derived SNP type in the Ames Ancestor and A0488 (Vollum) genomes respectively. The CDC 684 isolate creates a new branch (51 SNPs in length) midway along the Vollum branch and shares this node with 15 isolates that were obtained from a collection from the CDC [22].

were 15 isolates that shared the Vollum branch node with CDC 684. These isolates were predominantly recovered by the Centers for Disease Control during the 1950s and 1960s. While the incidence of lethal anthrax infections in the United States had been greatly reduced during the $20^{\text {th }}$ century [21], it can be assumed that the majority of the CDC isolates labeled as B. anthracis would have come from sources containing virulent strains such as imported hides and/or animal deaths [22].

Table 1 lists 27 non-synonymous chromosomal SNPs from 51 total that are unique to CDC 684 in comparison to the Vollum (A0488) strain. There are no obvious B. cereus or B. anthracis virulence factors on this list but the role for each of these proteins in CDC 684 may also be compromised by the large inversion event. It also needs to be reiterated that while these SNPs are unique in their relationship to the Vollum strain their status in 15 other un-sequenced isolates who shared the node along the Vollum branch are still undetermined. It is likely that most of these SNPs will be shared (i.e., no differences) with these 15 presumably virulent $B$. anthracis isolates.

Sequence variations between the virulence plasmids The simplest explanation for the attenuated phenotype for CDC684 would be the mutation of one or more of the 
Table 1 CDC 684 specific non-synonymous SNPs indicating chromosomal positions, gene products and amino acid changes

\begin{tabular}{|c|c|c|c|c|}
\hline Gene & Position & Product & $A-a a$ & $D-a a$ \\
\hline GBAA0382 & 47140 & ABC transporter, substrate binding & W & * \\
\hline GBAA0414 & 435861 & Hypothetical protein & 1 & V \\
\hline GBAA0492 & 488260 & Amino Acid permease family & A & V \\
\hline GBAA0715 & 734957 & ABC phosphate binding protein & Q & * \\
\hline GBAA0925 & 935719 & Putative lipoprotein & $E$ & G \\
\hline GBAA1301 & 1248597 & PAP 2 family protein & S & $\mathrm{N}$ \\
\hline GBAA1858 & 1742404 & Major facilitator transporter & V & G \\
\hline GBAA2173 & 2023504 & Conserved hypothetical protein & G & D \\
\hline GBAA2372 & 2212248 & Non-ribosomal peptide synthetase & V & A \\
\hline GBAA2649 & 2470526 & Putative permease & M & V \\
\hline GBAA2936 & 2704543 & Putative membrane protein & $P$ & S \\
\hline GBAA4328 & 3953215 & Conserved hypothetical protein & A & $\mathrm{T}$ \\
\hline GBAA4328 & 3953216 & Conserved hypothetical protein & A & V \\
\hline GBAA4353 & 3973954 & Acetylglutamate kinase & G & R \\
\hline GBAA4388 & 4002699 & Phosphate butyryl transferase & A & V \\
\hline GBAA4408 & 4021751 & Acetyl-CoA carboxylase & Q & * \\
\hline GBAA4430 & 4037923 & Hypothetical protein & A & $\mathrm{T}$ \\
\hline GBAA4515 & 4109405 & RNA polymerase sigma-43 & K & $\mathrm{R}$ \\
\hline GBAA4516 & 4110631 & DNA primase & $P$ & $L$ \\
\hline GBAA4521 & 4113151 & CBS domain protein & A & V \\
\hline GBAA4733 & 4305747 & ABC-transporter, ATP-binding & G & $\mathrm{R}$ \\
\hline GBAA4890 & 4443754 & Thiol peroxidase & $\mathrm{N}$ & S \\
\hline GBAA5207 & 4726424 & Conserved hypothetical protein & $\mathrm{T}$ & M \\
\hline GBAA5377 & 4870264 & SpoVA family protein & I & V \\
\hline GBAA5530 & 5021797 & Conserved hypothetical protein & $E$ & K \\
\hline GBAA5678 & 5165710 & ABC transporter, ATP-binding & A & $\mathrm{T}$ \\
\hline GBAA5703 & 5193199 & RNA helicase, DEAD/DEAH box & A & V \\
\hline
\end{tabular}

virulence factors encoded on the $\mathrm{pXO} 1$ or $\mathrm{pXO} 2$ plasmids that altered expression or function. These virulence factors include the toxin gene complex on pXO1 (comprising genes encoding for protective antigen, edema factor, and lethal factor), the poly-D-glutamyl capsule gene complex on pXO2 (encoded by $\operatorname{cap} A, \operatorname{cap} B, \operatorname{cap} C$ and $a c p A$ ), and trans-acting transcription regulators on both plasmids [23]. However, in silico comparison of the completed sequences of the pXO1 and pXO2 plasmids from the CDC 684 strain to those of the Ames Ancestor and Vollum strains showed that all of the known virulence factors were intact. There was a single non-synonymous SNP found in pXO1 GBAA_pXO1_0019, a large gene of unknown function. Collectively we observed no putative functional differences in the plasmid-encoded virulence factors between CDC 684 and its closest relative, Vollum, which is a fully virulent strain [24].

\section{Large chromosomal inversion in CDC 684}

The most striking feature of CDC 684 genome is a massive inversion that reverses the orientation of $3.3 \mathrm{Mbp}$ of the chromosome relative to the replication origin. The dimensions of the inversion have been graphically illustrated in a recent review of Bacillus anthracis genome variation [25]. This earlier report used Artemis software http://www.sanger.ac.uk/resources/software/ artemis/to illustrate the alignment and conserved gene order of four finished and closed genomes (B. anthracis Ames, B. anthracis Australia 94, B. anthracis CDC 684, and $B$. thuringiensis $\mathrm{Al}$ Hakam). While the fine-scale gene order in CDC 684 is precisely maintained as in the Ames chromosome, the large rearrangement has caused an inversion of a $3.3 \mathrm{Mbp}$ region between the basepair coordinates $454 \mathrm{Kbp}$ and 3,783 Kbp in the Ames Ancestor chromosome (see Figure 2).

The inversion appears to have been caused by an internal recombination event between homologous regions within two lysogenic lambda-like prophages (LambdaBa04 and LambdaBa02), which are found in all B. anthracis genomes $[26,27]$. The inversion can best be visualized at the molecular level by examining the orientation of the att (attachment) sites that flank the ends of these phages (Figure 2). Lysogenic bacteriophages possess cohesive ends (att), usually 12-13 bp repeats, which serve as both excision points and "sticky ends" that enable the phage to cirularize as it enters a lytic life cycle [28]. At first glance it seemed likely that the inversion may involve the att sites in these Lambda like prophages and that the exchange may have involved a site-specific recombination. But the two att sites were unique to each other, i.e., Lambda Ba04 and $\mathrm{Ba} 02$ contain distinct att sites (Figure 2B) that allow them to be distinguished from each other (Ba04, ATACAGCTCATGT and Ba02, TTTT(C/T)TTTACAC). In Ames Ancestor, pairs of these two distinct att sites define both the size $(\mathrm{Ba} 04=37.3 \mathrm{~kb} ; \mathrm{Ba} 02=44.0 \mathrm{~kb})$ and boundaries of each prophage. In CDC 684 (Figure 2A), the external att sites (represented by black bars) are in relatively identical chromosomal positions to those in the Ames Ancestor. However, the internal att sites (represented by green and red bars) have dramatically exchanged positions between these genomes. In CDC 684, the right att site (red bar) for LambdaBa04 has moved to the left att position of Lambda Ba02, and likewise the left att site for Lambda Ba02 (green bar) has moved to the position occupied by right att site in Lambda Ba04. The net effect of this exchange is the creation of new hybrid prophages in CDC 684 (Figure 2B). These observations indicate that the large inversion event did not involve site-directed recombination but rather a homologous recombination event in the interior of both prophages.

Molecular detection of the inversion in other B. anthracis strains

A PCR approach was designed to detect the inversion sites in CDC 684 as a method that could test for the 


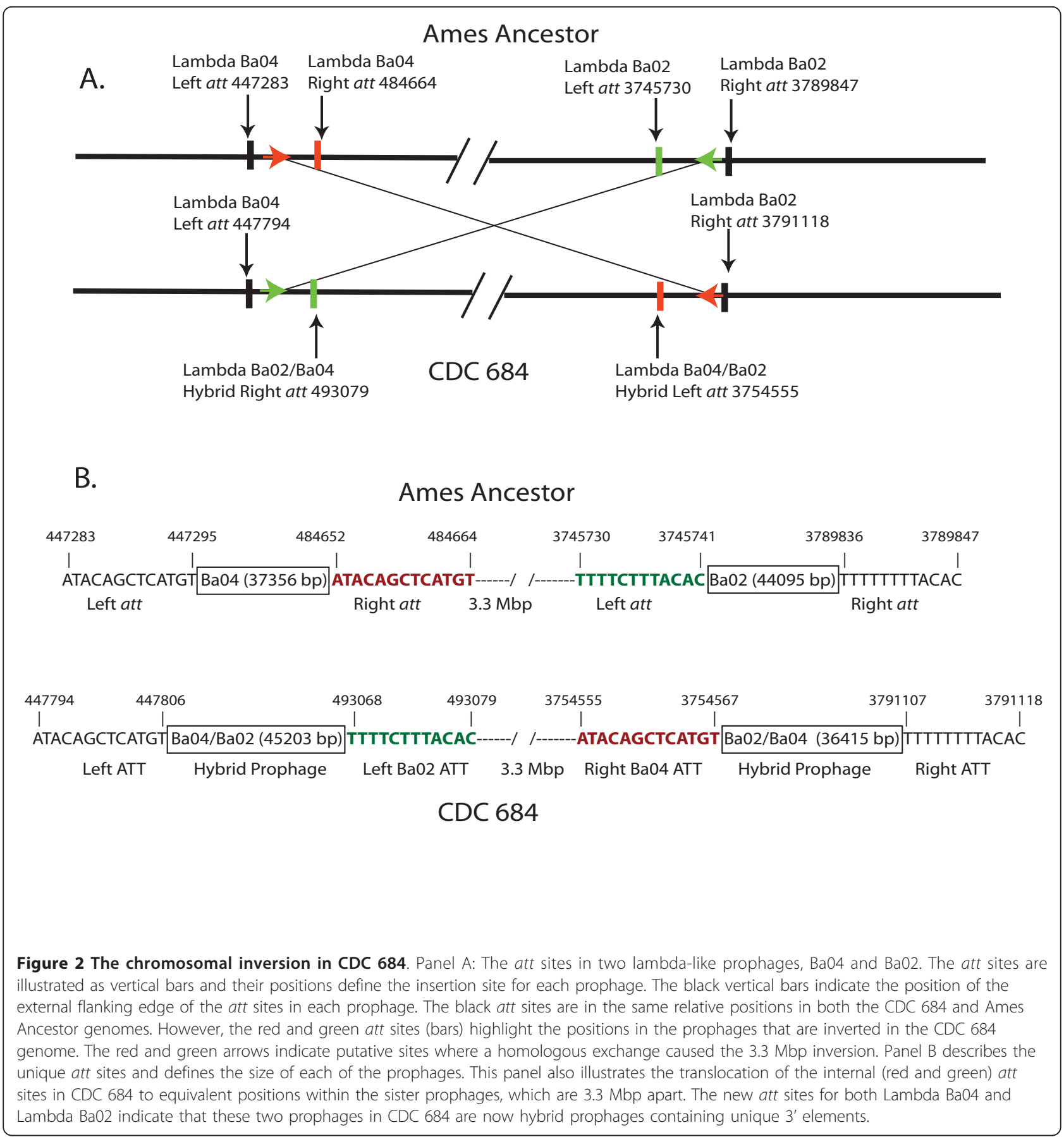

presence of the inversion in other isolates. Because of its size, the inversion is readily visible in the closed genome, but the molecular nature of the inversion is dependent on the proper alignment of two short regions (i.e. $165 \mathrm{bp}$ ) during the assembly of this genome. As illustrated in Figure 3, the 5' end of each of the rep sequences are distinct from each another and their positions are fixed at approximately the same positions in both genomes. However, the 3' end of the rep genes are highly homologous, with scattered SNPs the only distinguishing feature between these paralogs.

Due to constraints on PCR amplicon size we used mismatch amplification mutation assays (MAMA, [29]) to discriminate between the right and left ends of the large inversion in CDC 684 and Ames Ancestor. The rationale was to demonstrate the different ends of the inverted 3.3 Mbp fragment in CDC 684 by use of real time PCR assays. The MAMA system was designed to 


\section{Lambda Ba04 rep protein (GBAA_0438)}

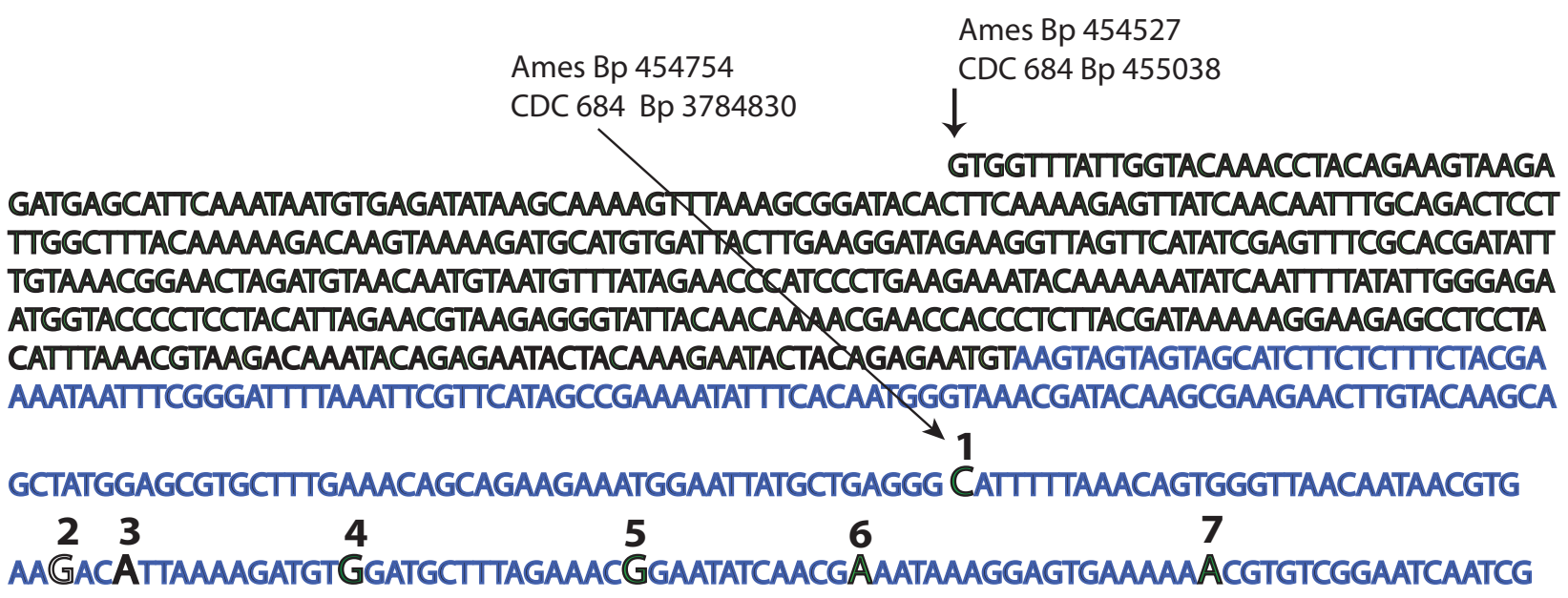

GAAGAGTGATGACTCGGATAGTGAATACATCGGCTTGTAG

3.3 Mbp

—CTACAAGCCGATGTATTC 7" 6" 5"

AC TATCCGAGTCATCACTCTTCCGATTGATTCCGACACGC TTTTTCACTCCTTTATIGCGTTGATATTCAGTTTCTAAAG

4" 3" 2"

CATCAACATCTITTAAA GTTTCACGTTATTGTTAACCCACTGTTTAAAATACCCTCAGCATAATTCCATTTCTCTGC

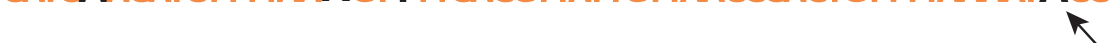

TGTTTCAAAGCACGCTCCATAGCTGCTTGTACAAGTTCTTCGCTTGTATCGTITACCATTGTGAAATATTTTCGGCTATG

AACGAATTTAAAATCCCGAAATTATTITCGTAGAAAGAGAAGATGCTACTACTACTITGTATATCAGTACTTAGTTCTTTAG

TATTTAGTTCTTTIAGTACTTAGTAGCTCGGGATTTTCCACCGGTGGTTITTCCACTGGTGGCTTTTICCGCCACTGGGTCATT TTGTGGAACTTCATATATGATTGTTTCCCACTTAATAATTTTGTTGTTATCATCTCTCACTGGAAATCTTTTAAGATACCCATA TTCCTTTAGTTCTTTCATTCCAGCTCTTAAGCTATCTAGCCCATCCTTTGCATGCCTAGATAATTQTTCTCTATAAAAAAACC AATCATCAGGAAGTGTAAGAATATACGCTAAAATCCCTTTTGCTTTCCAACTTAATCTTTTIATCTITIAGACCTGTATTATTT ATTGTCGTGTAGTTCTTATCTITATTAACTCGAAAAGTAGCCAT

Ames Bp 3784117
CDC 684 Bp 3785388

Ames Bp 3783424

CDC 684 Bp 455263

\section{Lambda Ba02 rep protein (GBAA_4120)}

Figure 3 Site of the CDC 684 inversion. This figure contains the entire sequences for the Lambda Ba04 and Lambda Ba02 rep genes. The 5 nucleotides in black represent regions unique to the prophage rep genes and are in the same directionality in the CDC 684 and Ames Ancestor genomes. The blue and orange nucleotides represent the $3^{\prime}$ ends that are homologous to both rep genes, and likely represent the site of recombination that resulted in the $3.3 \mathrm{Mbp}$ inversion in CDC 684. Seven SNPs that define the prophage 3' ends are in larger font, and are labeled 1-7 or 7"-1" for Lambda Ba04 or Lambda Ba02, respectively. In CDC 684, the allele states for these prophages have switched chromosomal positions relative to Ames Ancestor.

take advantage of polymorphic differences that characterize the left and right SNP signatures within the rep Lambda-like protein sequences relative to the Ames Ancestor genome. Both the left and right assay systems have common primers (CP, Table 2 and Methods) that are fixed because they are external to the $3.3 \mathrm{Mbp}$ inversion site. The internal primers are nearly identical but they target mismatches at specific SNP sites; G on the left site and $A$ on the right site of the Ames genome.
Table 2 MAMA assays used to detect the CDC 684 chromosomal inversion

\begin{tabular}{lcl}
\hline Left Inversion Primers & Polymorphism & Assay Targets \\
\hline CP Left-inv-F + Right-inv-F & A & CDC 684 \\
CP Left-inv-F + Left-inv-R & G & All other B. anthracis \\
\hline Right Inversion Primers & Polymorphism & Assay Targets \\
\hline Left-inv-R + CP Right-inv-R & G & CDC 684 \\
Right-inv-F + CP Right-inv-R & A & All other B. anthracis \\
\hline Where CP = Common Primer; inv = Inversion; F = Forward; R = reverse
\end{tabular}


These same internal primers (e.g., CP-Left-Inv-F and Left-Inv-R, Table 2) cannot amplify the same 400 and 500 bp products in CDC 684 because they are separated by $3.3 \mathrm{Mbp}$. But the reciprocal pairings of the internal primers do amplify products from CDC 684.

These MAMA were used to analyze several isolates within the Vollum branch. In addition, the SNPs flanking the inversion were compared to in silico analysis of other B. anthracis WGS to determine the configuration of this $3.3 \mathrm{Mbp}$ region in other non-Vollum strains. Table 3 illustrates that only the CDC 684 isolate possessed the inverted genotype from among 17 genomes examined, indicating the inversion is not common in $B$. anthracis.

\section{Defining the dif site in $B$. anthracis}

In $E$. coli the large ter region has been found to contain a specific substrate sequence, dif (for Deletion Induced Filamentation), which is used by two recombinases, XerC and XerD, to resolve chromosomal multi-mers and to allow daughter chromosomes to segregate before cell division [30,31]. It has been proposed that the dif site (a short palindromic sequence) is in fact a more likely site of termination than any specific ter sites for both the $E$. coli and B. subtilis chromosomes [32]. From the perspective of the CDC 684 genome, the dif sites in both $\gamma$-proteobacteria and Firmicutes appear to have an extremely close association with the maximum GC-skew in those genomes that have been analyzed [32,33].

Dif sites have been defined in both B. subtilis [34] and a member of B. cereus sub-group [32]. A cursory survey of

Table 3 Status of the Large Inversion Site by PCR or in silico analysis of 18 B. anthracis genomes

\begin{tabular}{llll}
\hline Isolate & Lineage & Assay & Orientation \\
\hline A0488 & Vollum & Real-time PCR & Ames-like \\
A1136 & Vollum & Real-time PCR & Ames-like \\
A1093 & Vollum & Real-time PCR & Ames-like \\
A1094 & Vollum & Real-time PCR & Ames-like \\
A0363 & Vollum & Real-time PCR & Ames-like \\
A0474 & Vollum & Real-time PCR & Ames-like \\
CDC 684 & Vollum & Real-time PCR & CDC 684 \\
A0493 & W.N.A. & In silico & Ames-like \\
A0442 & Kruger B & In silico & Ames-like \\
A0402 & CNEVA & In silico & Ames-like \\
Tsiankovskii & A.Br.008/009 & In silico & Ames-like \\
A0174 & W.N.A. & In silico & Ames-like \\
A0465 & CNEVA & In silico & Ames-like \\
A0389 & A.Br.001/002 & In silico & Ames-like \\
A0193 & W.N.A. & In silico & Ames-like \\
A2012 & Ames & In silico & Ames-like \\
A0248 & Aust 94 & In silico & Ames-like \\
A1055 & C-Branch & In silico & Ames-like \\
\hline
\end{tabular}

the palindrome from the $B$. subtilis and B. cereus dif site (AATATATATT) in the Ames Ancestor identified a 28bp palindromic sequence [32] that is located at nearly the precise genomic site of the cumulative GC-skew. This sequence is conserved and positioned at the cumulative $\sim 180^{\circ} \mathrm{GC}$-skew position of every complete whole genome sequence in all of the GenBank entries for the $B$. cereus sub-group (Table 4). The one exception is the genome of CDC 684 where the conserved dif-like sequence and the GC-skew are oriented at $\sim 120^{\circ}$ in relationship to the origin of replication (Figure 4, Table 4).

\section{Growth Kinetics of CDC 684 versus wild type B. anthracis}

The significant difference in the spatial orientation of the ori site and dif/GC skew sites in CDC 684 suggests that there could be an alteration in how the bi-directional replication of chromosome would proceed because of the unequal distances the opposite leading strands would need to travel. Because accumulated evidence indicates that genomes like those of $E$. coli and Bacillus sp do not tolerate significant changes between the spatial orientation of the ori and ter sites, we designed a growth experiment to compare the growth kinetics of CDC 684 to those of three wild type $B$. anthracis strains.

Growth curves for four strains of Bacillus anthracis: Ames, Ba_A0361 (a B branch isolate), Vollum and CDC 684 were grown in $\mathrm{LB}$ broth at $37^{\circ} \mathrm{C}$ (Figure 5). These cultures were grown in duplicate (Ames, BaA0361) or triplicate (Vollum, CDC 684) with growth measured by $\mathrm{OD}_{600}$. The strains represent two major phylogenetic groups of $B$. anthracis. Note the relatively consistent growth curves for the three wild type isolates: Ames, Ba

Table 4 Chromosomal locations of GC-skew, dif sites and their relative orientation in relationship to the Origin of Replication in complete genomes.

\begin{tabular}{lllll}
\hline Isolate & Genome & GC Skew & dif Position & ${ }^{*}$ Orientation \\
\hline Bc biovar Ba Cl & 5196054 & 2514865 & 2516079 & $174^{\circ}$ \\
Bc 03BB102 & 5269628 & 2587080 & 2592759 & $177^{\circ}$ \\
BcQ1 & 5214195 & 2507935 & 2510631 & $171^{\circ}$ \\
BCAH187 & 5269030 & 2560736 & 2564400 & $174^{\circ}$ \\
BC AH820 & 5301683 & 2566169 & 2575244 & $174^{\circ}$ \\
BC B4264 & 5419036 & 2617378 & 2620353 & $174^{\circ}$ \\
BC E33L & 5300915 & 2570501 & 2571014 & $174^{\circ}$ \\
BC ATCC 14579 & 5411809 & 2673035 & 2681358 & $178^{\circ}$ \\
BC ATCC 10987 & 5224283 & 2585881 & 2590339 & $178^{\circ}$ \\
BC G9842 & 5387334 & 2591148 & 2591275 & $173^{\circ}$ \\
Ba Ames Ances. & 5227419 & 2498507 & 2507867 & $172^{\circ}$ \\
CDC 684 & 5230115 & 1720671 & 1732304 & $119^{\circ}$ \\
Bt 97-27 & 5237682 & 2529472 & 2560322 & $173^{\circ}$ \\
Bt Al Hakam & 5257091 & 2591702 & 2593007 & $177^{\circ}$ \\
Bt BMB171 & 5330088 & 2601041 & 2608011 & $176^{\circ}$ \\
\hline
\end{tabular}



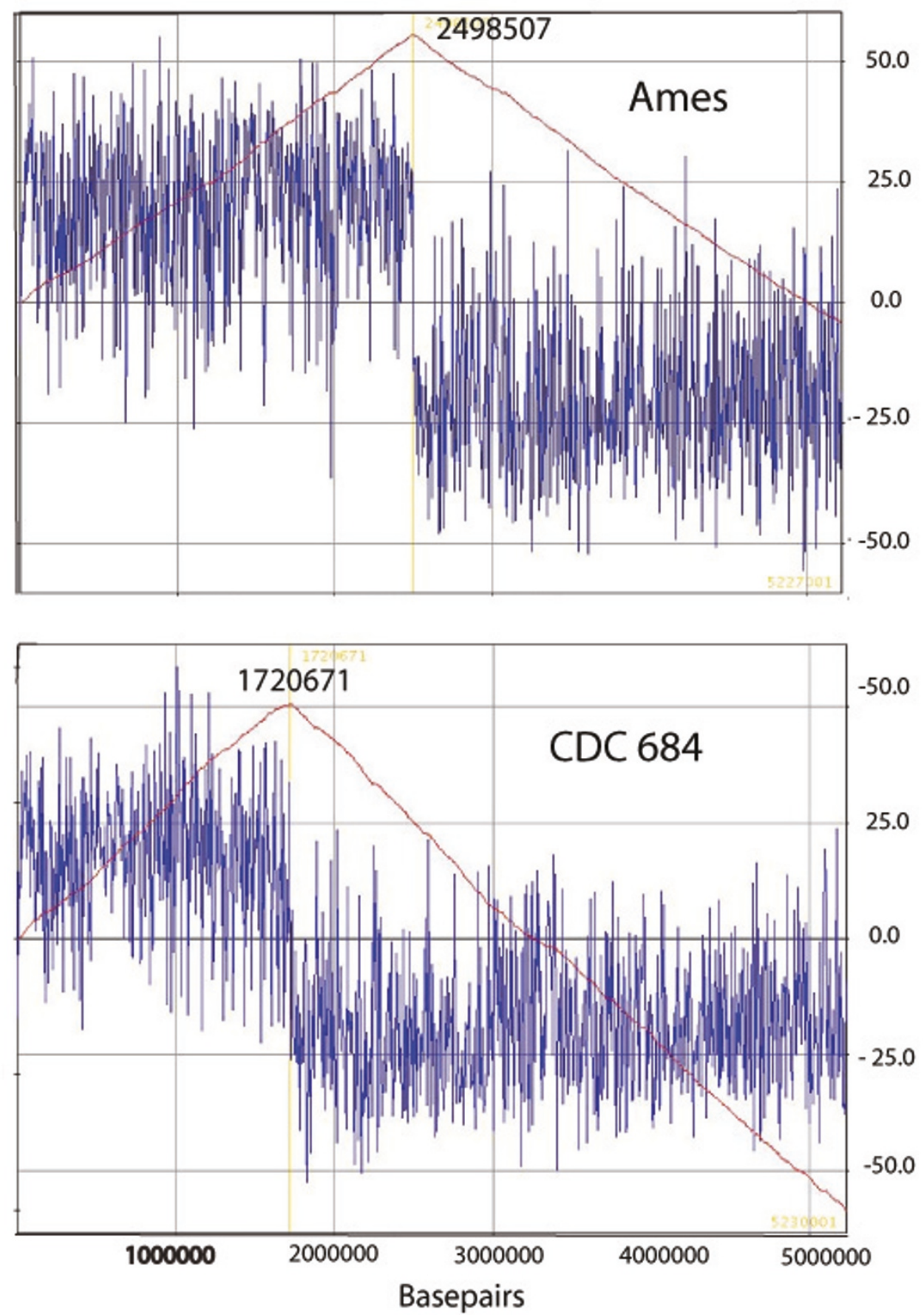

Figure 4 GC Skew Plot for B. anthracis Ames and B. anthracis CDC 684. GenSkew http://genskew.csb.univie.ac.at/, was used to compute the cumulative GC skew for these two complete genomes. Note that the position denoting the maximum skew for CDC 684 has shifted dramtically in comparison to the Ames Ancestor genome. This suggests that the terminus of replication for CDC 684 may be in an altered positon.

A0361 and Vollum, the closest relative to CDC 684. Two obvious differences between the CDC 684 and Vollum growth curves is a longer lag phase and slower mid $\log$ growth rate in CDC 684. These differences were noted despite careful efforts to exactly match inoculum sizes using direct plating viability counts. An extended lag phase would suggest that CDC 684 takes longer to adapt to the inoculum transfer process and/or to 


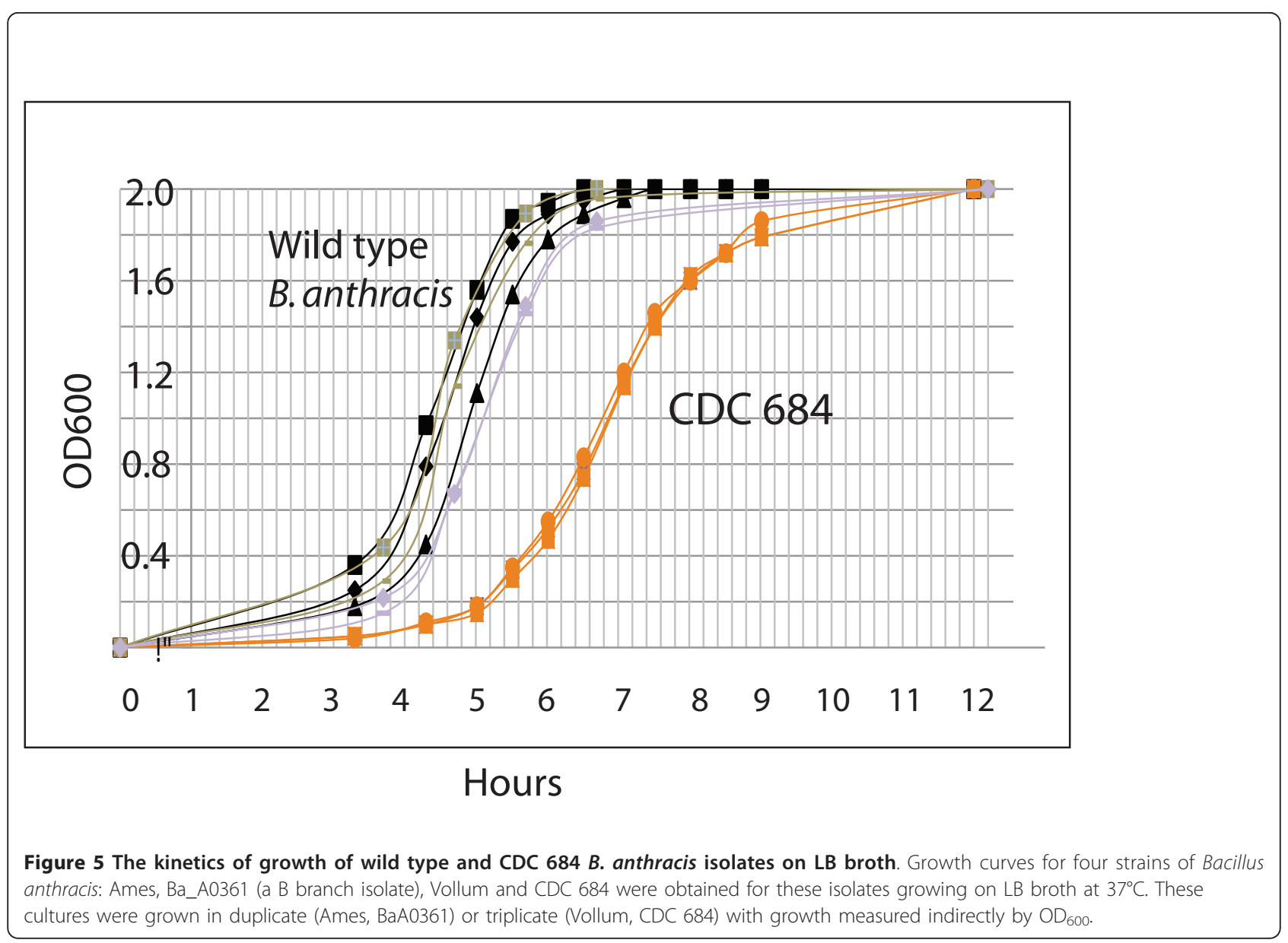

conditions necessary for growth and cell division. The slower mid log growth rate ( $\sim 55 \mathrm{~min}$ in Vollum and $\sim 80 \mathrm{~min}$ in CDC684) in this experiment suggests that even after revival from lag phase that CDC 684 has a cellular limitation to growth that does not exist in the wild type strains. These results provide a growth parameter that implies that the spatial change in the orientation of the origin of replication and the termination site in CDC 684 may have altered the growth of this isolate.

\section{Discussion}

By phylogenetic, molecular and clinical criteria, CDC 684 is a $B$. anthracis and its attenuated phenotype must be due to differences within its genome relative to those of other closely related $B$. anthracis strains. The marked degree of attenuation of CDC 684, (with an LD50 of $>1 \times$ $10^{8}$ spores by the i.m. route in the guinea pig) compares with LD50 values of 175 and 306 spores reported for the virulent Ames and Vollum-1B strains [16,17]. It therefore renders a comparative genomics approach highly informative and suggests that either subtle SNP differences and/or a dramatic and massive inversion within this chromosome are responsible for the attenuation.
Whole genome sequencing and comparative analysis indicates that there are 51 chromosomal and $<6$ plasmid SNP that are unique to CDC 684 in a comparison to Vollum. The possibility that one or more of these rare SNPs may have an important role in the attenuation of CDC 684 remains a viable option. These data have defined a new CDC 684 lineage emanating from the original Vollum branch, Figure 1. Twenty-seven of these SNPs would be translated into non-synonymous mutations in putative gene functions. None of these SNPs, however, are in genes considered to be virulence factors found in opportunistic $B$. cereus pathogens that include a variety of hemolysins, non-hemolytic enterotoxins, monomeric entertoxins and phospholipases [35]. The remaining 23 SNPs include 11 synonymous SNPs, 7 SNPs in pseudogenes, and 5 intra-genic SNPs. Only one of these intra-genic SNPs is located in a region within a promoter region $(-7 \mathrm{bp})$ in a L-serine dehydratase gene (GBAA_4361).

What has not been excluded from this new lineage are $15 \mathrm{~B}$. anthracis isolates that currently share the nodal position between the CDC 684 and Vollum lineages (see Figure 1). A sequencing effort to identify CDC 684 
specific SNP that are either shared or still unique among the presumably virulent 15 isolates would point to phenotype altering SNP. Any chromosomal and plasmid SNP that are still unique to CDC 684 would be candidates for having positions in genes or regulatory regions with roles that govern known or unknown functions that are necessary in a virulent organism. There is, as yet, no clear notion whether or how any of these SNPs could cause the dramatic change in the virulence or growth properties of CDC 684.

The role of the chromosome of B. anthracis in the overall etiology of the disease anthrax is still poorly understood. It is becoming evident that the regulatory functions of the virulent plasmids (pXO1 and pXO2) work in concert with certain chromosomal regulatory functions in a virulent organism, e.g. the regulation of the pXO1 atxA gene by chromosomal sigma factors or plasmid genes involved in a signal-transduction pathway that inhibits sporulation [36]. These and other recent studies [37] make it difficult to dismiss any of the CDC 684 non-synonymous mutations as candidates for a role in the attenuated phenotype without further analysis.

An alternative hypothesis to explain the attenuation of CDC 684 is a role for the large $3.3 \mathrm{Mbp}$ inversion within its chromosome. While this inversion does not appear to have altered the fine-scale order of the individual genes, it has changed the orientation of the genes within the inversion with respect to the genes outside of the inversion. This change in the orientation has been illustrated by whole genome alignments [25] and by an analysis of the GC skewing and the location of dif sites of the CDC 684 genome and that of several $B$. anthracis and B. cereus sub-group isolates (Figure 4, Table 4). These analyses indicate that the spatial relationship between the origin of replication and the termination of replication in CDC 684 has been perturbed by the massive inversion. The comparative growth data (Figure 5) clearly supports the idea that chromosomal replication may be altered in CDC 684 by exhibiting an extended lag phase and a longer growth rate.

The longer DNA synthesis time needed to complete chromosomal replication may be sufficient, alone, to explain the slower cellular growth rate of CDC 684. In the asymmetrical CDC 684 chromosome, the longer leading strand distance is $3.783 \mathrm{Mbp}$ vs. $2.615 \mathrm{Mbp}$ for Vollum and all characterized wild type $B$. anthracis strains. This is a $\sim 38 \%$ larger chromosomal distance to replicate and, assuming everything else remains constant, this will take that much longer to complete the entire chromosome. The mid log doubling time difference between the wild type strains ( $80 \mathrm{~min})$ and CDC 684 ( $~ 80 \mathrm{~min})$ is $\sim 45 \%$. The similarity between the $38 \%$ long replication distance and $45 \%$ longer growth rate is striking. This observation suggests that the displaced ter region remains the site for replication termination and that the asymetrical longer leading strand replication distance in CDC 684 becomes limiting for growth in vitro.

Historical accounts suggest that there are strong tendencies to conserve the basic relationship between the position of the ori and ter sites in enteric bacteria [38]. Following the discovery of the dif sites and related specific recombinases, it was proposed that the topological relationship between the ori and the ter/dif site must be maintained at $0^{\circ}$ and $180^{\circ}$, respectively, for normal chromosomal segregation to occur [31]. This was suggested because mutations in the Xer recombinase genes or the dif site or the displacement of the dif site to other regions of the chromosome had adverse effects on cell division.

More recently whole genome sequence comparisons between several distinct species also suggest that there is conservation in the spatial orientation between the ori and ter sites over broad groups of bacteria [39-41]. Dot plots of conserved DNA and protein sequences between pairs of species produce characteristic X-shaped patterns suggesting that large chromosomal rearrangements often revolve around and maintain the distances between the origin and the terminus.

This study illustrates a case where the naturally conserved $180^{\circ}$ orientation of the ori and ter sites has been modified by a large chromosomal inversion in a strain of $B$. anthracis, CDC 684. We suggest that the consequence of the altered spatial relationship between the ori and ter sites from $180^{\circ}$ to $120^{\circ}$ has caused the change in growth kinetics of this isolate (Figure 5). We also suggest that this change appears to alter the length of time that CDC 684 takes to replicates its chromosome. Whether this change has also altered the virulent phenotype of this isolate is yet to be determined.

\section{Conclusions}

We address two hypotheses that could have a role for the attenuated phenotype in B. anthracis CDC 684. The first is that a single chromosomal point mutation may have altered a function that is crucial to normal growth and virulence in $B$. anthracis.

Despite evidence for a wide array of chromosomal rearrangements in the $B$. cereus subgroup [42], BLAST searches conducted using the dif region of Bacillus anthracis and B. cereus also indicate a trend towards maintaining a nearly $180^{\circ}$ spatial relationship between the ori/dif sites $\left(171^{\circ}\right.$ - to $178^{\circ}$, Table 4$)$. The second hypothesis, therefore, suggests that major alterations of this relationship are possibly not tolerated by $B$. anthracis when under natural ecological pressures. The existence of an isolate like CDC 684 demonstrates that a moderate skewing in the spatial relationship between 
the ori / dif may be overcome in terms of sheer growth and survivorship in the laboratory. But we suggest that the potential biological consequences of altered DNA replication and/or DNA expression rendered by this change may have resulted in an altered phenotype for successful pathogenicity in a mammalian host. Both kinds of "genetic alterations" can be expected to be rare in $B$. anthracis since these organisms would not have a selective advantage in a natural environment and would be difficult to find.

\section{Methods}

\section{Whole genome sequencing and assembly}

The genome of $B$. anthracis CDC 684: Chromosome [GenBank: CP001215.1]. pXO1 [GenBank: CP001216] and pXO2 [GenBank: CP001214] was sequenced at the Joint Genome Institute (JGI)/J. Craig Venter Institute using a combination of $3 \mathrm{~kb}$ and $8 \mathrm{~kb}$ DNA libraries. All general aspects of library construction and sequencing performed at the JGI can be found at http://www. jgi.doe.gov/. Draft assemblies were based on 59,691 total reads. The Phred/Phrap/Consed software package http://www.phrap.com was used for sequence assembly and quality assessment $[43,44]$. After the shotgun stage, reads were assembled with parallel Phrap (High Performance Software, LLC). Possible mis-assemblies were corrected with Dupfinisher [45] or transposon bombing of bridging clones (Epicentre Biotechnologies, Madison, WI). Gaps between contigs were closed by editing in Consed and by custom primer walking (Roche Applied Science, Indianapolis, IN). A total of 1955 additional custom PCRs were necessary to close gaps and to raise the quality of the finished sequence. The completed genome sequence of $B$. anthracis str. CDC 684 contains 62,606 reads, achieving an average of 10 -fold sequence coverage per base with an error rate of $<10^{-6}$.

\section{Experimental animals and spore challenges}

Spores were prepared from B. anthracis CDC 684 as previously described [16] and female Hartley guinea pigs (660 g) were challenged intramuscularly (i.m.) with various spore concentrations (see 'Results') at USAMRIID as previously described $[16,46]$. Research was conducted in compliance with the Animal Welfare Act and other federal statutes and regulations relating to experiments involving animals and adheres to principles stated in the Guide for the Care and Use of Laboratory Animals (National Research Council. 1996. Guide for the care and use of laboratory animals National Academy Press, Washington, DC.). The facility where this research was conducted is fully accredited by the Association for Assessment and Accreditation of Laboratory Animal Care International.

\section{Canonical SNP (canSNP) Analysis}

The thirteen canSNP alleles and the specific assays for each have been described previously [19]. TaqMan ${ }^{\mathrm{TM}}$ Minor Groove Binding (MGB) allelic discrimination assays were used to determine the precise canSNP grouping for every isolate used in this study $[19,47]$.

\section{SYBR MAMA Assays}

Additional SNP genotyping was conducted using the Mismatch Amplification Mutation Assay [MAMA] [29], which is based on allele-specific PCR kinetics [48], enhanced by penultimate mismatch primer design $[29,49]$. The MAMA approach was also used to distinguish the inverted 3.3 Mbp segment of CDC 684 from all other $B$. anthracis strains. MAMA assays were designed for both the 5' (left) and 3' (right) ends of the inversion; i.e., two sets of primer products separated by 3.3 Mbp. The sequences flanking the $3.3 \mathrm{Mbp}$ inverted region were unique and common to both CDC 684 and the Ames genomes and were defined as Common Primers (CP). But the internal primers targeted nearly identical sequences and therefore used primers designed around mismatches that could distinguish and generate 400 and $500 \mathrm{bp}$ PCR products. The primers were as follows (5' to 3'): Left-inv-R (TAAAGCATCCACATCTTT-

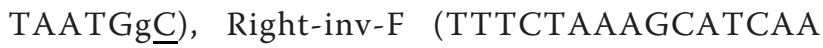
CATCTTTTAAAGgT), and CP-Left-inv-F (GCATGTGATTACTTGAAGGATAGAAGG) were used to characterize the left inversion, and Left-inv-R, Right-inv-F and CP-Right-inv-R (5'- AGATTTCCAGTGAGAGATGATAACAACA) targeted the right inversion. Underlined nucleotides overlap the SNP; nucleotides in lowercase represent deliberate penultimate mismatches. The two consensus primers contained no SNPs or incorporated mismatches. Expected inversion genotypes using these primers are listed in Table 2 in the Results section and an example of this assay system is illustrated in Additional File 1.

The MAMA assay system was also used to type 10 new canSNP sites that further define the Vollum lineage of $B$. anthracis. The primers for these sites are shown in Additional File 2 as a Table.

Each inversion SYBR MAMA reaction comprised $1 \mathrm{X}$ SYBR Green Master Mix (Applied Biosystems, Foster City, CA), 0.1 uM MAMA primer, $0.2 \mathrm{uM}$ consensus primer, $0.08 \mathrm{U}$ Platinum Taq polymerase (Invitrogen, Carlsbad, CA) and molecular grade $\mathrm{H}_{2} \mathrm{O}$ to $9 \mathrm{uL}$. One $\mathrm{uL}$ of genomic DNA was added to each well to a final volume of $10 \mathrm{uL}$. Reactions were carried out in 384well optical plates (Applied Biosystems) on an ABI Prism 7900 HT real-time instrument (Applied Biosystems) using the following thermocycling parameters: 2 min at $50^{\circ} \mathrm{C}, 10 \mathrm{~min}$ at $95^{\circ} \mathrm{C}$, followed by 50 cycles of $15 \mathrm{~s}$ at $95^{\circ} \mathrm{C}$ and $1 \mathrm{~min}$ at $60^{\circ} \mathrm{C}$. PCR products were 
subject to post-amplification dissociation $\left(15 \mathrm{sec}\right.$ at $95^{\circ}$ C, $15 \mathrm{sec}$ at $60^{\circ} \mathrm{C}, 15 \mathrm{sec}$ at $95^{\circ} \mathrm{C}$ ) to confirm product specificity.

Additional File 1 provides an example of real time PCR profiles for the left inversion region using a fixed Common Primer (CP) that is located outside of the left boundary of the $3.3 \mathrm{Mbp}$ inversion site in both CDC 684 and the Ames genomes. This figure demonstrates real time PCR kinetics for the detection of amplicons for the left boundary of the inversion site in both CDC 684 and the Ames Ancestor Genome using primer combinations described in Table 2.

\section{GC Skew Analysis}

A free software program, GenSkew http://genskew. csb.univie.ac.at/, was used to compute the cumulative skew for 15 complete WGS of B. anthracis, B. cereus and $B$. thuringiensis. These WGS data were downloaded from GenBank: http://www.ncbi.nlm.nih.gov/ genbank/.

\section{Growth Data}

Stocks of B. anthracis Ames, B. anthracis Vollum (A0488), B anthracis A0361 (a B branch isolate), and B. anthracis $\mathrm{CDC} 684$ were subcultured and grown for $\sim 19$ hours on LB agar. These cells were harvested and normalized to $\mathrm{OD}_{600}$ densities that correspond to $10^{5}$ $\mathrm{cfu} / \mathrm{mL}$ based on viable count estimates from previous experiments for each isolate. These measurements were used to precisely add $10^{5}$ cfu inoculums to create $3 \mathrm{ml}$ culture tubes for each isolate. These cultures were grown at $37^{\circ} \mathrm{C}$ and $\mathrm{OD}_{600}$ measurements were determined on a CO800 Spectrophotometer.

\section{Additional material}

Additional file 1: Figure S1: MAMA real time PCR. Example of MAMA Real Time PCR to Differentiate between a 3.3 Mbp inverted configuration in CDC 684 and the wild type configuration in Bacillus anthracis. The primers depicted in this figure were designed to detect the left configuration for the wild-type and/or the inverted sequence for the CDC 684 genome. The fixed common primer, CP Left-inv- $F$ was paired with both Right-inv-F and the Left inv-R primers. While the wild type Ames Ancestor can be amplified by the two LEFT inversion primers (Left inv $F$ and Left inv R); the left inversion site in CDC 684 can only be amplified by the CP left-inv-F and Right inv-F because the Right inversion forward site normally situated $3.3 \mathrm{Mbp}$ distal, has relocated to within $450 \mathrm{bp}$ of the CP left-inv-F primer. Note that these two panels represent samples with the same three primers and that the positive allele will amplify orders of magnitudes faster than the negative allele. The delta CT (cross-over threshold) values are the differences between a specific linear portion of the target sequence (in cycles) versus the number of cycles to obtain the same (threshold) signal in the non-target sequence (e.g....the delta CT for CDC 684 vs Ames is 13.4.

Additional file 2: Table S1: The SYBR MAMA primer sets for 10 Vollum lineage canSNP Assays

\section{Acknowledgements}

This work was funded in part by the Department of Homeland Security Science and Technology Directorate under contract numbers: NBCH2070001 and HSHQDC-08-C00158. Support for this project was also provide by NAU's Technology and Research Initiative Fund.

\section{Author details}

${ }^{1}$ Center for Microbial Genetics and Genomics, Northern Arizona University, Flagstaff, AZ 86011, USA. ${ }^{2}$ Bioscience Division, Los Alamos National Laboratory, Los Alamos, NM 87545, USA. ${ }^{3}$ Bacteriology Division, United States Army Medical Research Institute of Infectious Diseases, Fort Detrick, Frederick, MD 21702-5011, USA. ${ }^{4}$ Pathogen Genomics Division, Translational Genomics Research Institute, Phoenix, AZ 85004, USA.

\section{Authors' contributions}

All authors have read and approved the final version of the manuscript. RTO contributed to the interpretation of data and in generating the original draft of the manuscript. EPP made substantial contributions in design of experiments, acquistion and interpretation of data and in the critical editing of the manuscript. SRW made substantial contributions in acquisition and interpretation of data. JMG made substantial contributions in data acquired using bioinformatic tools and includes the visualization of the inversion. WKC was responsible for the generation of canSNP data and analysis. TP made substantial contributions in the development of specific markers and in phylogenetic analysis of CDC 684 and its relationship to the world-wide distribution of B. anthracis. GX was an important part of the Genome Sequencing team from Los Alamos who generated, completed and annotated the CDC 684 WGS. CM was an important part of the Genome Sequencing team from Los Alamos who generated, completed and annotated the CDC 684 WGS. KKH provided the impetus to acquire the CDC 684 isolate, confirmed the $B$. anthracis like genotype, initiated the eventual WGS of this isolate and provided a critical review and edit of the draft manuscript. JC provided the genome announcement and initial annotation of CDC 684 WGS as part of the Genome Sequencing team from Los Alamos. $\mathrm{BEI}$ conducted the animal spore challenges in Guinea pigs that demonstrated the attenuated phenotype of CDC 684. JMS provided comparative whole genome analysis of CDC 684 against other whole genome sequences and the identification of SNPs that are unique to the CDC 684 genome. SMB provided comparative whole genome analysis of CDC 684 against other whole genome sequences and the identification of SNPs that are unique to the CDC 684 genome. AF provided the animal spore challenge data and the interpretation of this data as well as a critical review of the initial draft of this manuscript. PK provided the inspiration and the major intellectual content for this work, was the first to describe the large inversion event and provided the first and final critical reviews of this document

Received: 11 May 2011 Accepted: 30 September 2011 Published: 30 September 2011

\section{References}

1. Sternbach G: The history of anthrax. J Emerg Med 2003, 24(4):463-467.

2. Tigertt WD: Anthrax. William Smith Greenfield, M.D., F.R.C.P., Professor Superintendent, The Brown Animal Sanatory Institution (1878-81). J Hyg Camb 1980, 85:415-420.

3. Sterne M: The immunization of laboratory animals against anthrax. Onderstepoort J Vet SciAnim Ind 1939, 13:313-317.

4. Makino S, Sasakawa C, Uchida I, Terakado N, Yoshikawa M: Cloning and $\mathrm{CO} 2$-dependent expression of the genetic region for encapsulation from Bacillus anthracis. Mol Microbiol 1988, 2(3):371-376.

5. Mikesell P, Ivins B, Ristroph J, Dreier T: Evidence for plasmid-mediated toxin production in Bacillus anthracis. Infection and immunity 1983, 39(1):371.

6. Okinaka RT, Cloud K, Hampton O, Hoffmaster AR, Hill KK, Keim P, Koehler TM, Lamke G, Kumano S, Mahillon J, et al: Sequence and organization of pXO1, the large Bacillus anthracis plasmid harboring the anthrax toxin genes. J Bacteriol 1999, 181(20):6509-6515.

7. Robertson D, Bragg T, Simpson S, Kaspar R, Xie W, Tippetts M: Mapping and characterization of the Bacillus anthracis plasmids. In Proceedings of the International Workshop on Anthrax. Edited by: Turnbull P. Salisbury, UK Salisbury Medical Society; 1990:55-58. 
8. Robertson DL, Leppla SH: Molecular cloning and expression in Escherichia coli of the lethal factor gene of Bacillus anthracis. Gene 1986, 44(1):71-78.

9. Tippetts MT, Robertson DL: Molecular cloning and expression of the Bacillus anthracis edema factor toxin gene: a calmodulin-dependent adenylate cyclase. J Bacteriol 1988, 170(5):2263-2266.

10. Vodkin MH, Leppla SH: Cloning of the protective antigen gene of Bacillus anthracis. Cell 1983, 34(2):693-697.

11. Welkos S, Lowe J, Eden-McCutchan F, Vodkin M, Leppla S, Schmidt J: Sequence and analysis of the DNA encoding protective antigen of Bacillus anthracis. Gene 1988, 69(2):287-300

12. Hoffmaster A, Hill K, Gee J, Marston C, De B, Popovic T, Sue D, Wilkins P, Avashia S, Drumgoole R: Characterization of Bacillus cereus isolates associated with fatal pneumonias: strains are closely related to Bacillus anthracis and harbor B. anthracis virulence genes. Journal of clinical microbiology 2006, 44(9):3352..

13. Hoffmaster AR, Ravel J, Rasko DA, Chapman GD, Chute MD, Marston CK, De BK, Sacchi CT, Fitzgerald C, Mayer LW, et al: Identification of anthrax toxin genes in a Bacillus cereus associated with an illness resembling inhalation anthrax. Proc Natl Acad Sci USA 2004, 101(22):8449-8454.

14. Klee S, Ozel M, Appel B, Boesch C, Ellerbrok H, Jacob D, Holland G, Leendertz F, Pauli G, Grunow R: Characterization of Bacillus anthracis-like bacteria isolated from wild great apes from Cote d'Ivoire and Cameroon. Journal of bacteriology 2006, 188(15):5333.

15. Ezzell J Jr, Abshire T, Little S, Lidgerding B, Brown C: Identification of Bacillus anthracis by using monoclonal antibody to cell wall galactose$\mathrm{N}$-acetylglucosamine polysaccharide. Journal of clinical microbiology 1990, 28(2):223..

16. Ivins B, Welkos S, Knudson G, Little S: Immunization against anthrax with aromatic compound-dependent (Aro-) mutants of Bacillus anthracis and with recombinant strains of Bacillus subtilis that produce anthrax protective antigen. Infection and Immunity 1990, 58(2):303.

17. Ivins $B E$, Fellows PE, Nelson GO: Efficacy of a standard human anthrax vaccine against Bacillus anthracis spore challenge in guinea-pigs. Vaccine $1994,12: 872-874$.

18. Pearson T, Busch JD, Ravel J, Read TD, Rhoton SD, U'Ren JM, Simonson TS, Kachur SM, Leadem RR, Cardon ML, et al: Phylogenetic discovery bias in Bacillus anthracis using single-nucleotide polymorphisms from wholegenome sequencing. Proc Natl Acad Sci USA 2004, 101(37):13536-13541.

19. Van Ert MN, Easterday WR, Huynh LY, Okinaka RT, Hugh-Jones ME, Ravel J, Zanecki SR, Pearson T, Simonson TS, U'Ren JM, et al: Global genetic population structure of Bacillus anthracis. PLoS One 2007, 2(5):e461.

20. Manchee RJ, Broster MG, Melling J, Henstridge RM, Stagg AJ: Bacillus anthracis on Gruinard Island. Nature 1981, 294(5838):254-255.

21. Brachman PS: Inhalation anthrax. Ann N Y Acad Sci 1980, 353:83-93.

22. Marston CK, Hoffmaster AR, Wilson KE, Bragg SL, Plikaytis B, Brachman P, Johnson S, Kaufmann AF, Popovic T: Effects of long-term storage on plasmid stability in Bacillus anthracis. Appl Environ Microbiol 2005, 71(12):7778-7780.

23. Koehler TM: Bacillus anthracis genetics and virulence gene regulation. Curr Top Microbiol Immunol 2002, 271:143-164.

24. Price L, Hugh-Jones M, Jackson P, Keim P: Genetic diversity in the protective antigen gene of Bacillus anthracis. Journal of bacteriology 1999, 181(8):2358

25. Keim P, Gruendike JM, Klevytska AM, Schupp JM, Challacombe J, Okinaka R: The genome and variation of Bacillus anthracis. Mol Aspects Med 2009, 30(6):397-405.

26. Read TD, Peterson SN, Tourasse N, Baillie LW, Paulsen IT, Nelson KE, Tettelin H, Fouts DE, Eisen JA, Gill SR, et al: The genome sequence of Bacillus anthracis Ames and comparison to closely related bacteria. Nature 2003, 423(6935):81-86

27. Sozhamannan S, Chute MD, McAfee FD, Fouts DE, Akmal A, Galloway DR, Mateczun A, Baillie LW, Read TD: The Bacillus anthracis chromosome contains four conserved, excision-proficient, putative prophages. BMC Microbiol 2006, 6:34.

28. Prescott LM, Harley JP, Klein DA: Microbiology. Dubuque, IA: Wm. C. Brown Publishers; , Third 1996.

29. Cha R, Zarbl H, Keohavong P, Thilly W: Mismatch amplification mutation assay (MAMA): application to the $\mathrm{cH}$-ras gene. Genome Research 1992, 2(1):14.
30. Blakely G, Colloms S, May G, Burke M, Sherratt D: Escherichia coli XerC recombinase is required for chromosomal segregation at cell division. New Biol 1991, 3(8):789-798.

31. Kuempel P, Henson J, Dircks L, Tecklenburg M, Lim D: dif, a recAindependent recombination site in the terminus region of the chromosome of Escherichia coli. The New biologist 1991, 3(8):799.

32. Hendrickson $\mathrm{H}$, Lawrence JG: Mutational bias suggests that replication termination occurs near the dif site, not at Ter sites. Molecular Microbiology 2007, 64(1):42-56.

33. Carnoy C, Roten C-A: The dif/Xer Recombination Systems in Proteobacteria. PLOS ONE 2009, 4(9):e6531.

34. Yen M, Lin N, Hung C, Choy K, Weng S, Tseng Y: oriC region and replication termination site, dif, of the Xanthomonas campestris pv. campestris 17 chromosome. Applied and environmental microbiology 2002, 68(6):2924.

35. Sergeev N, Distler M, Vargas M, Chizhikov V, Herold KE, Rasooly A: Mlcroarray analysis of Bacillus cereus group virulence factors. Journal of Microbiological Methods 2006, 65:488-502.

36. Perego M, Hoch JA: Commingling regulatory systems following acquisition of virulence plasmids by Bacillus anthracis. Trends Microbiol 2008, 16(5):215-221.

37. Chiang C, Bongiorni C, Perego M: Glucose-dependent activation of Bacillus anthracis toxin gene expression and virulence requires the carbon catabolite protein CcpA. J Bacteriol 2011, 193(1):52-62.

38. Liu SL, Sanderson KE: The chromosome of Salmonella paratyphi A is inverted by recombination between $\mathrm{rnnH}$ and rrnG.J Bacteriol 1995, 177(22):6585-6592.

39. Eisen JA, Heidelberg JF, White O, Salzberg SL: Evidence for symmetric chromosomal inversions around the replication of origin in bacteria. Genome Biology 2000, 1(6):1-9.

40. Tillier ER, Collins RA: Genome rearrangement by replication-directed translocation. Nat Genet 2000, 26(2):195-197.

41. Worning P, Hensen LJ, Hallin PF, Staerfeldt H-H, Ussery DW: Origins of replication in circular prokaryotic chromosomes. Environmental Microbiology 2006, 8(2):353-361.

42. Carlson CR, Kolsto AB: A complete physical map of a Bacillus thuringiensis chromosome. J Bacteriol 1993, 175(4):1053-1060.

43. Ewing B, Green P: Base-calling of automated sequencer traces using phred. II. Error probabilities. Genome Res 1998, 8(3):186-194.

44. Ewing B, Hillier L, Wendl MC, Green P: Base-calling of automated sequencer traces using phred. I. Accuracy assessment. Genome Res 1998, 8(3):175-185

45. Han CS, Xie G, Challacombe JF, Altherr MR, Bhotika SS, Brown N, Bruce D, Campbell CS, Campbell ML, Chen J, et al: Pathogenomic sequence analysis of Bacillus cereus and Bacillus thuringiensis isolates closely related to Bacillus anthracis. J Bacteriol 2006, 188(9):3382-3390.

46. Ivins BE, Ezzell JW Jr, Jemski J, Hedlund KW, Ristroph JD, Leppla SH: Immunization studies with attenuated strains of Bacillus anthracis. Infect Immun 1986, 52(2):454-458.

47. Morin PA, Saiz R, Monjazeb A: High-throughput single nucleotide polymorphism genotyping by fluorescent $5^{\prime}$ exonuclease assay. Biotechniques 1999, 27(3):538-540, 542, 544 passim.

48. Huang MM, Arnheim N, Goodman MF: Extension of base mispairs by Taq DNA polymerase: implications for single nucleotide discrimination in PCR. Nucleic Acids Res 1992, 20(17):4567-4573.

49. Rhodes RB, Lewis K, Shultz J, Huber S, Voelkerding KV, Leonard DG, Tsongalis GJ, Kephart DD: Analysis of the factor V Leiden mutation using the READIT Assay. Mol Diagn 2001, 6(1):55-61.

doi:10.1186/1471-2164-12-477

Cite this article as: Okinaka et al: An attenuated strain of Bacillus anthracis (CDC 684) has a large chromosomal inversion and altered growth kinetics. BMC Genomics 2011 12:477. 